\title{
Historia de la enfermedad de Chagas en Argentina: evolución conceptual, institucional y política
}

\section{The history of Chagas' disease in Argentina: conceptual, institutional, and political evolution}

\author{
Juan Pablo Zabala \\ CONICET - Consejo Nacional de Investigaciones Científicas \\ y Técnica; Universidad Nacional de Quilmes, \\ Av. Rivadavia, $2358,6^{\circ}$. Buenos Aires, Argentina \\ jpzeta@hotmail.com \\ Recibido para publicación en abril de 2009. \\ Aprobado para publicación en mayo de 2009.
}

ZABALA, Juan Pablo. Historia de la enfermedad de Chagas en Argentina: evolución conceptual, institucional y política. História, Ciências, SaúdeManguinhos, Rio de Janeiro, v.16, supl.1, jul. 2009, p.57-74

\section{Resumen}

La enfermedad de Chagas ha sufrido, a lo largo de sus cien años de reconocimiento, profundas modificaciones en su concepción científica, en su reconocimiento institucional y en su valoración política. Así, desde un punto de vista médico, se la ha concebido como causante del bocio, se han resaltado sus efectos agudos, y luego sus efectos sobre la salud cardiaca. Del mismo modo, la política sanitaria ha sucesivamente descartado la importancia de la enfermedad, luego elevado a nivel de una causa nacional y paulatinamente dejado en el margen de las agendas. El presente artículo presenta, de un modo resumido, los puntos centrales de esa trayectoria histórica en Argentina, dando cuenta de los aspectos cognitivos, políticos e institucionales que dieron sustento a la enfermedad, entendida como un hecho a la vez social y biológico.

Palabras clave: enfermedad de Chagas; Argentina; historia de las enfermedades.

Abstract

In the one hundred years since the identification of Chagas disease, major changes have occurred in its scientific conception, institutional recognition, and political weight. From a medical perspective, it was seen as the cause of goiter, next its acute effects were emphasized, and then its effects on cardiac health received greater attention. In similar fashion, sanitary policy first downplayed the disease's importance, then elevated it to the role of a national cause, and gradually relegated it to the bottom of the agenda. The article briefly presents the key points of this historical trajectory in Argentina, exploring the cognitive, political, and institutional underpinnings of the disease as both a social and biological fact.

Keywords: Chagas' disease; Argentina; history of disease. 
A cien años de su descubrimiento, la enfermedad de Chagas afecta en América Latina a alrededor de 8 millones de personas, y constituye una amenaza para otros 28 millones (Guhl, Lazdins-Helds, 2007). Pero no solo la cantidad de infectados define la importancia del problema: en la medida en que su reproducción está asociada a condiciones materiales de pobreza, escasez de información y falta de acceso a servicios sanitarios básicos, la enfermedad es un símbolo de las profundas inequidades que arrastra el continente.

La gravedad de la situación ha sido reconocida, en modo explícito o implícito, por distintas esferas de la sociedad, tanto en el plano nacional como internacional, al punto que el tema fue objeto de diferentes planes de política sanitaria y científica desde la década de 1950. En Argentina, estas medidas incluyeron la creación de instituciones destinadas a llevar adelante tareas de control, medición y atención de la enfermedad, así como el desarrollo de programas específicos de apoyo a la investigación científica sobre el tema. Estas iniciativas, aunque resultaron insuficientes para erradicar la enfermedad, han asegurado una densidad de actores sociales articulados alrededor de las prácticas de producción de conocimientos científicos y de intervención asociadas a ella.

A pesar de la continuidad que puede sugerir su persistencia, la trayectoria de la enfermedad de Chagas ha sido, al menos en Argentina, particularmente cambiante. Considerando que una enfermedad es una entidad de múltiples dimensiones (biológica, social, cognitiva, institucional y política ${ }^{1}$ ), las profundas redefiniciones en estos planos condicionaron fuertemente la manera en que la enfermedad se conformó, a lo largo de su historia, como entidad mórbida, como objeto de salud pública y como tema de investigación científica. De hecho, durante el siglo XX han cambiado radicalmente los síntomas atribuidos a la enfermedad, las formas de diagnóstico, el tipo de actores involucrados, los espacios de producción de conocimientos, la consideración de los enfermos e incluso el tipo de instituciones dedicadas a su atención.

En este trabajo analizo las relaciones entre estas dimensiones, e intento mostrar cómo la trayectoria de la enfermedad de Chagas estuvo fuertemente ligada a los contextos políticos e institucionales a los que pertenecían los actores que impulsaron su reconocimiento, a las disciplinas científicas que se erigieron en cada momento como las principales productoras de conocimientos (y de formas de intervención), a las circunstancias políticas que excedieron ampliamente los espacios de definición de la enfermedad (los cambios de gobierno, los golpes de Estado, las persecuciones políticas), y las iniciativas surgidas en el plano internacional, entre otros factores.

El análisis pone énfasis en el ámbito de la producción de conocimientos científicos. Este ha sido uno de los espacios donde el 'mal de Chagas' ha recibido una atención constante desde la década de 1930, y en las última décadas, por diferentes motivos (disponibilidad de fondos para estudiar el tema, compromiso político de los investigadores con el desarrollo del país, dinámicas de reproducción de la comunidad académica), se ha convertido en un 'caso exitoso' de desarrollo científico en la periferia, en la medida en que esas investigaciones cuentan con un "amplio reconocimiento de su relevancia y legitimidad" por parte de la comunidad científica internacional (Coutinho, 1999, p.520). 


\section{2-1926: descubrimiento y negación de la enfermedad de Chagas}

El anuncio del descubrimiento de una nueva enfermedad, por parte de Carlos Chagas en Brasil en 1909, tuvo un pronto eco en Argentina. La existencia de similares condiciones de hábitat favorables a la transmisión, la presencia de los insectos vectores y el carácter endémico del bocio y cretinismo - supuestas manifestaciones de la etapa crónica de la enfermedad - en ciertas regiones del país hicieron pensar a los miembros del Instituto Nacional de Bacteriología - luego Instituto Bacteriológico - del Departamento Nacional de Higiene, en la posibilidad de que la enfermedad de Chagas también fuera una patología importante del norte de Argentina. De esta forma, la enfermedad pasó a formar parte de las inquietudes del Instituto, el principal espacio de estudios sobre las enfermedades de importancia epidemiológica, con el objetivo de "darle a la primera autoridad sanitaria nacional, las bases necesarias para el estudio de nuestros problemas relativos a la defensa de la salud humana" (Lozano, 1920, p. 16).

Sin embargo, en forma inversa a lo sucedido en Brasil, donde los trabajos de Carlos Chagas funcionaron como disparador a un conjunto de indagaciones destinadas a acumular evidencia científica sobre distintos aspectos relacionados con las características de la enfermedad (Stepan, 1981), las primeras investigaciones realizadas en Argentina tuvieron como consecuencia el cuestionamiento de la importancia de la enfermedad de Chagas y significaron, consecuentemente, un marcado desinterés por parte de la comunidad médica y de investigación sobre el tema durante las siguientes dos décadas.

\section{Las investigaciones en el Instituto Bacteriológico y la negación de la enfermedad}

Entre 1912 y 1916 se llevaron a cabo un conjunto de investigaciones (Rosenbusch, Maggio, 1912; Kraus, Maggio, Rosenbusch, 1915, 1916), lideradas por el bacteriólogo austríaco y director del Instituto Bacteriológico Rudolf Kraus, que tenían como objetivo dar cuenta de la presencia de enfermos crónicos de Chagas en el norte argentino. Sin embrago, el resultado de esos trabajos no arrojó datos coincidentes con el modelo de enfermedad propuesto por Chagas, que proponía que la acción del parásito en el organismo humano causaba bocio y cretinismo, constituyendo así los principales síntomas de la enfermedad en su 'etapa crónica'2. Por un lado, se comprobó la existencia de insectos vectores (vinchucas) infectados con el Trypanosoma cruzi, aunque no se pudieron encontrar rastros del parásito en los enfermos de bocio analizados (los supuestos casos crónicos de acuerdo a las formulaciones de Chagas). Por otro lado, la distribución de los parásitos y vectores no coincidía con la de los enfermos de bocio y cretinismo, lo que llevó a pensar que "la vinchuca infectada en la República Argentina con mucha probabilidad no produce la enfermedad de Chagas [probablemente debido a] una atenuación del tripanosoma por el clima" (Kraus, Maggio, Rosenbusch, 1916, p.177). Como conclusión, estas investigaciones descartaron la presencia de enfermos de Chagas crónicos en Argentina, donde las medidas de profilaxis no debían alterarse por los descubrimientos de Chagas ya que el "bocio y el cretinismo [locales] deben combatirse con las mismas medidas que el europeo" (p.178).

De este modo, durante las primeras décadas del siglo XX, la enfermedad de Chagas no fue considerada un problema de salud en la Argentina, como consecuencia de al menos 
tres factores. En primer lugar, por las limitaciones epistémicas que imponía la asociación entre bocio y T. cruzi, que llevaron a que, al descartar esta relación, haya sido negado el conjunto de la enfermedad, el menos en su etapa crónica. ${ }^{3}$ En segundo lugar, por el contexto institucional en el que las indagaciones fueron realizadas. En este sentido, siendo un órgano del Departamento Nacional de Higiene, el rol del Instituto Bacteriológico era dar cuenta de la magnitud y distribución de las enfermedades identificadas en el país, y eventualmente la producción de medios terapéuticos para su atención.

En este marco institucional, pues, la no comprobación fehaciente de la endemia relegó la enfermedad de Chagas a un espacio marginal frente a otras enfermedades que, como la malaria o la peste, no dejaban dudas acerca de su importancia. Por último, existían también limitaciones de orden técnico, ya que la identificación del parásito suponía procedimientos poco estabilizados y de difícil dominio: los métodos serológicos desarrollados por Guerreiro y Machado ${ }^{4}$ no eran de uso extendido (y aún así, tenían una muy baja especificidad). La observación directa de un $T$. cruzi mediante microscopio resultaba muy dificultosa, requiriendo un entrenamiento y dedicación muy distintas a lo requerido por otras enfermedades endémicas de la región, como la malaria, complejidad que se elevaba aún más en la etapa crónica de la enfermedad, incluso mediante las pruebas realizadas en las autopsias. Finalmente, las pruebas disponibles para detectar el parásito en forma indirecta (inoculación de animales sensibles ${ }^{5}$, xenodiagnóstico ${ }^{6}$ ) suponían un trabajo experimental considerable y no eran practicables a gran escala.

\section{7-1945: los comienzos de la investigación científica en Argentina - Salvador Mazza y el reconocimiento de la enfermedad}

Tras ser desechada su importancia epidemiológica, pasaron más de 10 años sin investigaciones sobre la enfermedad de Chagas en Argentina. ${ }^{7}$ El interés por la enfermedad volvió a resurgir, lentamente, cuando se encontraron los primeros casos de hombres y perros infectados con el parásito. Esto sucedió en el marco de dos misiones de investigadores extranjeros que, siguiendo la lógica de los 'cazadores de parásitos' (Worboys, 1993), realizaron incursiones científicas en el país, y aportaron nuevas técnicas que facilitaron la observación del parásito en la sangre de los humanos. La primera de ellas, en 1924, fue liderada por Peter Mühlens, del Institut für Tropenmedizin de Hamburgo, y la segunda, un año después, por Charles Nicolle (premio Nobel en 1929), del Institut Pasteur de Tunez (Mülhens, 1924; Mazza, 1926).

En buena medida gracias a la influencia de Nicolle, la Universidad de Buenos Aires creó, en 1926, la Misión de Estudios de Patología Regional Argentina (Mepra), un laboratorio ubicado en el norte del país y dedicado a la exploración y estudio de las enfermedades de la zona. El director de ese laboratorio fue uno de los discípulos de Nicolle, Salvador Mazza, un médico con una trayectoria ligada a la investigación y al trabajo de laboratorio y entrenado en bacteriología (entre otros, con el propio Nicolle). A diferencia de los investigadores del Instituto Bacteriológico, Mazza desarrolló un programa de investigaciones sobre las distintas enfermedades existentes en la región, más allá de su importancia epidemiológica, abarcando las dolencias más comunes como otras no registradas o poco 
habituales (entre otras, la leishmaniasis, la brucelosis, comúnmente llamada 'fiebre ondulante', y luego la enfermedad de Chagas). La aproximación a cada una de estas patologías no se limitaba a su diagnóstico y registro, sino que se pretendían hacer aportes al conocimiento general de la enfermedad, tanto de sus condiciones de infección como de las características de las manifestaciones clínicas de cada una de ellas (Mazza, 1939; Sierra Iglesias, 1990, p.164).

Dentro de este marco institucional y cognitivo, hacia 1933 se identificaron los primeros casos agudos de enfermedad de Chagas en Argentina (Romaña, 1934; Mazza, Ruchelli, 1934). A partir de allí, las actividades de la Mepra se dedicaron casi exclusivamente al estudio de esta enfermedad, de sus condiciones de transmisión, de su extensión, y de sus diferentes cuadros clínicos, llegándose en pocos años a identificar varios cientos de casos.

Estas investigaciones tuvieron como resultado un cambio fundamental en la concepción de la enfermedad, en al menos dos sentidos sustanciales. Desde el punto de vista político, la certificación de los casos colocó a la enfermedad - si bien de un modo incipiente dentro del mapa de intereses de la comunidad médica y, por extensión, de la salud pública del país; y desde un punto de vista cognitivo, implicó una redefinición de numerosos aspectos de la enfermedad que se encontraban en un estado controversial y que ponían en duda la incidencia epidemiológica de la enfermedad de Chagas (el número de casos certificados hacia 1930 no superaba los cincuenta en Brasil, y en toda América superaban escasamente la centena, de acuerdo con Yorke (1937, citado en Kropf, 2006), las condiciones efectivas de transmisión, el cuadro clínico de la enfermedad. ${ }^{8}$ Estas controversias tenían, resumidamente, dos ejes centrales: por un lado, la dudosa relación entre T. cruzi y bocio, y por otro, la falta de otros signos externos (descartando el bocio) que permitieran una comprobación de la infección en los humanos.

\section{Estrategias de investigación en torno a la enfermedad de Chagas}

La forma en que Mazza se dedicó a la enfermedad fue cualitativamente distinta a la de los otros investigadores que se habían aproximado al tema en el país (principalmente, los del Instituto Bacteriológico). Mientras que estos últimos estaban orientados a la identificación de los 'problemas de salud', Mazza tomó a la enfermedad como una línea de investigación sobre la que desarrolló buena parte de su carrera científica, menos condicionado por las presiones sanitarias. Esta diferencia implicó un cambio sustancial: mientras que en la década de 1920 las investigaciones se limitaron a la (no) comprobación de los enunciados de Chagas, Mazza se dedicó, una vez encontrado el parásito, a indagar los distintos aspectos sobre los que no existía evidencia concluyente (lo que lo llevó, en buena medida, a retomar las investigaciones en el punto en que las había dejado Carlos Chagas).

Así, pueden identificarse distintas etapas en los trabajos de Mazza, que dan cuenta de la manera en que la enfermedad se fue constituyendo como objeto de investigación. El primer paso fue, durante los primeros años de la década de 1930, la comprobación de animales infectados naturalmente con el T. cruzi, por ejemplo un perro (Mazza, 1926), distintas clases de armadillos, murciélagos o comadrejas (Mazza, 1939). 
Hacia 1933, con el hallazgo de los primeros casos de humanos infectados comenzó una nueva etapa, donde las investigaciones se orientaron a la elucidación de los efectos del parásito en las personas, concentrándose en la etapa aguda (cuando la infección es reciente), y sin abrir juicios definitivos sobre la etapa crónica (la relación con el bocio era aludida, aun por el propio Chagas, como "un elemento poco esclarecido" (Chagas, 1934, citado en Kropf, 2006). Los resultados de esas investigaciones lideradas por Mazza fueron centrales para la sistematización de la enfermedad en su etapa aguda, al punto de definir "un cuadro sintomático, que una vez conocido, es difícil confundir con el de otros procesos", caracterizado por presentar, "con excepción de signos de bocio, todos los otros síntomas característicos de la forma aguda de la tripanosomiasis" (Mazza, Ruchelli, 1934, p.3), que incluían, principalmente, fiebre, decaimiento y taquicardia como signos principales, y eventualmente, como signo externo la presencia de un edema bipalpebral de un ojo (inflamación de los párpados superior e inferior de un ojo).

La inflamación del ojo (Romaña, 1935), luego reconocida como 'signo de Romaña', fue un elemento central para el reconocimiento de la enfermedad por fuera de la reducida comunidad de especialistas (que al momento, además del grupo liderado por Mazza en Argentina, incluía a los sucesores del propio Chagas, como su hijo Evandro Chagas, Emmanuel Dias, entre otros). Y al mismo tiempo se transformó en un motivo de fuertes disputas entre Salvador Mazza (y sus colaboradores) y Cecilio Romaña (apoyado por los investigadores brasileros). Sintéticamente, la posición de Mazza restaba importancia a la inflamación ocular como hecho aislado. Es decir, si bien no negaba que esta inflamación - que según Mazza (1943) había sido señalada a Romaña por él mismo - era útil como elemento orientador de la pesquisa, lo integraba a un cuadro clínico más complejo y negaba originalidad a Romaña, ya que esos síntomas se encontraban descriptos, "desde un primer momento, por el descubridor de este padecimiento, Carlos Chagas" (Mazza, 1943). ${ }^{9}$

En cambio, Mazza sostenía que la difusión de la enfermedad se sustentaba, por un lado, en el entrenamiento de una extensa red de colaboradores, que lo proveían de los materiales que le permitieron acumular la cifra de casos (de hecho, Romaña fue inicialmente uno de sus colaboradores, y luego separado del plantel de la Mepra). Y por otro lado, a la estrategia de difusión a través de diferentes medios: algunos habituales, como las publicaciones (Mazza acumuló cerca de quinientas publicaciones en treinta años de carrera), y la participación en los distintos Congresos nacionales y extranjeros (sobre todo, cumpliendo roles en la organización), y otros menos comunes, como las charlas divulgativas a especialistas, que Mazza daba por toda la región del norte (Sierra Iglesias, 1990).

Siguiendo el patrón de trabajo señalado, Mazza había acumulado, hacia fines de la década de 1930, más de 1200 casos de enfermos agudos certificados. Gracias a esta evidencia, y a la iniciativa de Mazza en los distintos espacios profesionales, la enfermedad volvió a ser considerada, bajo sus nuevas formas, como una entidad de importancia por parte de las comunidades médicas argentina y brasilera. Al menos en el ámbito local, Mazza tuvo un reconocimiento acorde: hubo diversas propuestas para cambiar el nombre a 'enfermedad de Chagas-Mazza'10, y en Argentina ese fue el nombre oficial que adquirió la enfermedad, y adoptado y enseñado en las Universidades (Fonzo Gandolfo, 1939). En 1946, Mazza muere de una enfermedad cardíaca sin dejar discípulos a cargo de sus investigaciones, en 
medio de un período de transición de la Mepra, que fue trasladada a la ciudad de Buenos Aires y puesta bajo la dirección de médicos no especialistas en la enfermedad, y finalmente disuelta en 1959.

\section{5-1955: reconfiguración cognitiva y reformas de salud - la enfermedad de Chagas como 'problema social nacional'}

Entre fines de la década de 1940 y mediados de la década de 1950, la enfermedad de Chagas fue definitivamente reconocida como un problema sanitario y social de relevancia nacional, tanto por la extensión epidemiológica atribuida (los afectados pasaron a ser calculados en alrededor de 1 millón, sobre una población de menos de veinte millones de habitantes) como por los elementos simbólicos movilizados a su alrededor. ${ }^{11}$

$\mathrm{El}$ ascenso definitivo a la agenda de las autoridades sanitarias y al imaginario social estuvo fuertemente asociado a dos procesos de orígenes históricos independientes, pero que confluyeron en la redefinición de la enfermedad (tanto en sus dimensiones médicas, políticas y simbólicas). Por un lado, una reconfiguración en el plano cognitivo, que puso de relieve su condición de enfermedad crónica cardiaca de gran extensión epidémica; y por el otro, una profunda reforma en el plano de las políticas sanitarias que tuvo lugar en el gobierno de Juan Domingo Perón, encabezada por su ministro de Salud Ramón Carrillo, que tuvo como una de sus marcas principales la implementación de un conjunto de medidas de tipo sanitarista y de combate a enfermedades infecciosas.

En el plano cognitivo, tuvieron gran importancia las investigaciones llevadas a cabo por Cecilio Romaña, antiguo colaborador de Salvador Mazza), en el Instituto de Medicina Regional de la Universidad Nacional de Tucumán. Estas investigaciones tuvieron cambios centrales con respecto a las llevadas a cabo anteriormente: en primer lugar, pusieron el foco en los trastornos cardiacos de largo plazo que causaba la infección con el T. cruzi (Romaña, Cossio, 1944), en línea con los trabajos que se realizaban en Brasil (Dias, Laranja, Nóbrega, 1945; Laranja, Dias, Nóbrega, 1948) y en oposición al interés por la etapa aguda observada hasta ese momento. Por otro lado, incluyeron la utilización de cálculos estadísticos que proveyeron las primeras evidencias epidemiológicas sobre la extensión de la enfermedad en el país.

De esta forma favorecieron un nuevo cambio en la conceptualización de la enfermedad (de un mal agudo a un mal crónico ${ }^{12}$ ), y constituyeron la base sobre la que la enfermedad fue planteada como problema sanitario por parte del estado, pasando del millar de enfermos a alrededor de 1.000 .000 de infectados calculados, en base a un trabajo empírico limitado - alrededor de 1200 análisis realizados entre 1945 y 1951, con métodos variados que incluyeron el xenodiagnóstico y métodos serológicos -, y luego extrapolando el índice de infección al resto de la población que vivía en zonas de presencia del parásito (Romaña, 1953). ${ }^{13}$

Al mismo tiempo, con la redefinición de la enfermedad también se modificó el sujeto identificado como un 'enfermo chagásico', pasando de la población exclusivamente rural a todos aquellos que, producto de los movimientos migratorios, habitaban en las ciudades. 


\section{Peronismo, nacionalismo y reformas de salud: el sanitarismo de Ramón Carrillo}

El reconocimiento de la enfermedad como problema social estuvo fuertemente vinculado a otros procesos concomitantes con la producción de conocimiento científico novedoso. Así, fueron determinantes, en el plano político, los profundos cambios que hubo en el país a partir del gobierno de Juan Perón en 1946, y particularmente en las políticas de salud. ${ }^{14}$ En el plano internacional, fue central la existencia de procesos similares en varios países de América Latina, principalmente en Brasil y Chile, pero también en otros países como Ecuador; y por último, pero no menos importante, la disponibilidad de medios técnicos para la realización de campañas de profilaxis, basadas en la eliminación del agente transmisor de la enfermedad por medio del hexaclorociclohexano (Romaña, Ábalos, 1948; Dias, Pellegrino, 1948).

Las reformas en el área de salud incluyeron la creación, por primera vez en el país, de un Ministerio de Salud Pública, encargado de desarrollar políticas de alcance nacional. Estas reformas estuvieron fuertemente orientadas por el pensamiento sanitarista, definido por el propio Carrillo (1974, p.365) como "aquella rama de la salud publica que tiene por objeto organizar los medios técnicos, jurídicos, científicos para eliminar y destruir lo gérmenes patógenos y evitar e impedir, así, la contaminación del hombre, directa o indirectamente, protegiéndolo de las contingencias o injurias del medio ambiente".

Dentro de estas políticas, se destacaron las iniciativas de combate a un conjunto de enfermedades, entre las que se incluían el paludismo, la fiebre amarilla y la propia enfermedad de Chagas. En particular, esta fue además caracterizada como "un problema nacional", en el doble sentido de: (a) objeto de las políticas sanitarias de alcance nacional que se lanzaron en ese gobierno, y (b) objeto de conocimiento fuertemente impulsado por investigadores argentinos, básicamente por Mazza y Romaña, dejando de lado a los investigadores brasileños que habían continuado con las investigaciones sobre el tema, y con los que el propio Romaña tenía una relación estrecha (Carrillo, 1951, p.20).

En este contexto, y en buena medida gracias a las relaciones entre Carrillo y Romaña, se creó, en 1950, el Comité Directivo de Investigaciones y Profilaxis de la Enfermedad de Chagas, la primera institución encargada oficialmente de combatir la enfermedad, que dependía del flamante Ministerio de Salud de la Nación. El Comité fue la manifestación del inicio, en Argentina, de lo que su director, el propio Romaña (1951, p.215), denominaba "la era de los insecticidas de acción residual y [de] la 'nueva conciencia' despertada por la medicina preventiva".

En 1952 este Comité se convirtió en el Servicio Nacional de Profilaxis y Lucha contra la Enfermedad de Chagas (SNPLECH), también a cargo de Romaña, órgano "encargado de la aplicación, cumplimiento y vigilancia de los programas redactados para ese fin" (decreto 2177, 8 jul. 1952). En ese marco tuvieron lugar las primeras medidas de intervención sobre la enfermedad, siguiendo las consideraciones técnicas que surgían de los trabajos experimentales de Romaña, llevándose a cabo las primeras campañas de rociado con insecticida (gammexane) de un modo sistemático en la provincia de Chaco, La Rioja y Catamarca, aunque éstas sólo tuvieron un carácter experimental y un muy corto alcance de viviendas rociadas (Silveira, 2000). 


\section{6-1972: Ia institucionalización del problema social}

La dinámica social que, hacia mediados de la década 1950, había llevado a la enfermedad de Chagas a ser reconocida como problema de salud pública y como objeto de investigación científica se vio fuertemente afectada por el derrocamiento del gobierno de Perón en septiembre de 1955. Sin embargo, diferentes actores en nuevos contextos institucionales renovaron en los años siguientes el interés por la enfermedad, consolidando el proceso de institucionalización que se había iniciado tiempo atrás.

En el plano conceptual, durante la década de 1950 y 1960 prevalecieron las nociones acerca de la enfermedad de Chagas que se estabilizaron durante la década de 1940 y principios de 1950, esto es, como una enfermedad cardiaca crónica, causada por el T. cruzi y transmitida por las vinchucas, asociada a las condiciones de pobreza de las viviendas rurales, y con una importante extensión epidemiológica. En ese marco, como consecuencia de la asociación entre la infección y el desarrollo de cardiopatías crónicas, en el plano cognitivo, y de los nutridos movimientos migratorios desde las zonas rurales hacia los centros urbanos, el tema se instaló en los servicios de atención de salud de las ciudades, y con ello surgió un nuevo mapa de actores e instituciones. Se produjo un proceso de 'urbanización' de la enfermedad: ya no se trataba solamente de poblaciones rurales afectadas, sino de enfermos urbanos con discapacidad crónica (y por ende laboral). De esta forma, la enfermedad implicaba no solo padecimientos a quienes la sufrían, sino que también comenzaron a considerarse los perjuicios ocasionados al estado, tanto por los gastos que ocasionaba la atención de los enfermos como por la pérdida de la fuerza laboral del país.

La consolidación de la enfermedad de Chagas como un problema social de primer orden que se observó en el período que sigue al gobierno de Perón estuvo marcada por la creación de una serie de instituciones destinadas a su identificación, medición y control de la transmisión. Durante estos años, el SNPLECH creado por el ministro Carrillo fue creciendo y transformándose hasta convertirse en el Instituto Nacional de Parasitología Fatala Chabén, dedicado casi exclusivamente al diagnóstico de la enfermedad de Chagas; se creó el Programa Nacional de Chagas, una estructura nacional dedicada a la fumigación de las viviendas rurales; y se sancionaron una serie de normas de regulación de las transfusiones sanguíneas, para evitar otra forma importante de transmisión de la enfermedad (Indiech, 1995).

Este desarrollo institucional tuvo consecuencias tanto sobre la producción de conocimientos vinculados a la enfermedad, como sobre las relaciones entre los referentes del campo científico y los procesos de toma de decisiones en el plano político. A diferencia de los períodos anteriores, donde las dinámicas institucionales estaban estrechamente ligadas a las trayectorias de ciertos sujetos, a partir de fines de la década de 1950 el espacio social vinculado a la enfermedad se vuelve mucho más denso y complejo. El proceso de institucionalización de la enfermedad implicó que las prácticas de los actores se fueran autonomizando crecientemente de las circunstancias personales de sus protagonistas, y sobrevivió a los importantes avatares políticos que atravesó el país entre el fin de la década de 1950 y principios de los años $1970 .^{15}$ 
El desarrollo de estas instituciones (Programa Nacional de Chagas, Laboratorio Fatala Chabén) influyó fuertemente sobre la relación entre la producción de conocimientos y las instancias de reconocimiento e intervención sobre la enfermedad. Si en las épocas anteriores fueron ciertos miembros de la comunidad científica quienes lideraron los procesos de reconocimiento de la enfermedad (Mazza en la Mepra y Romaña desde el IMR), en este período la producción de conocimiento quedó subsumida en la estructura institucional en la que se tradujo el interés por el problema social. De hecho, fue en el marco de estas instituciones vinculadas a la salud pública, tanto aquellas dedicadas exclusivamente al Chagas como los hospitales públicos, donde se produjo la mayor parte de los conocimientos vinculados a la enfermedad, lo que marcó un cambio importante con respecto a los períodos anteriores. En el plano conceptual, la producción de conocimiento estuvo, durante este período, estrechamente ligada a los problemas que imponía el aparato institucional vinculado a la enfermedad: la identificación, la cuantificación y la interrupción del contagio.

Resumiendo, el período que va del derrocamiento de Perón hasta principios de la década de 1970 se caracterizó por la traducción del problema en una serie de nuevas instituciones que tuvieron a la atención de la enfermedad como principal misión y como elemento legitimador (tanto frente a las demás agencias del estado como al interior de la comunidad profesional de sus miembros). Las principales actividades relacionadas con la enfermedad, así, pasaron a formar parte de las misiones institucionales de estas nuevas organizaciones (fumigación, aprobación de medicamentos, control de donación de sangre).

\section{2-1985: la conformación de un espacio de investigación vinculado al Chagas}

En los años de 1970 y principios de la década de 1980, se observó una relativa estabilización de las iniciativas destinadas a controlar la enfermedad iniciadas en los años 1960, y con ello una definitiva incorporación del Chagas en diversas instituciones del país (Universidades, centros de atención, organismos encargados de reglamentar las políticas sanitarias y laborales). Estas medidas permitieron, aunque con ciertos altibajos, disminuir la cantidad de infectados en el país, pasando de un promedio del 10,1 del total de la población para el período 1964-1969 a un promedio del 5,8 en 1981 (Segura, 2002).

En el plano de la investigación científica, en cambio, la consideración sobre la enfermedad dio un vuelco sustancial, y se convirtió en un objeto largamente atendido por la comunidad de investigadores del país (y de otros países de América Latina, como Brasil). Así, se dio en estos años un significativo crecimiento de la investigación científica sobre el tema, en distintos aspectos relacionados con la enfermedad. Este vuelco obedeció al menos a dos motivos principales: el interés por incorporar a la investigación científica algunos nuevos de relevancia social; y la existencia de financiamiento sostenido, a través del Programa Nacional de Investigación de Enfermedades Endémicas de la Secretaría de Estado de Ciencia y Tecnología (SECyT), creado en 1973, y del Programa Especial de Investigación y en Enfermedades Tropicales (TDR) de la Organización Mundial de la Salud (OMS), a partir de 1975.

La principal consecuencia que esto tuvo fue la redefinición de la relación entre los espacios de producción de conocimientos científicos y las instancias de definición e intervención sobre la enfermedad de Chagas. Esta nueva relación se caracterizó, principalmente, 
por una apuesta a la investigación científica como una estrategia de intervención legítima sobre la enfermedad, a través del desarrollo de nuevas drogas o vacunas que permitieran tratamientos más efectivos que los existentes hasta el momento, que nunca lograron desarrollarse. De esta forma, la producción de conocimiento científico perdió el papel casi exclusivo de brindar las herramientas técnicas necesarias para el diagnóstico y cuantificación de la enfermedad. En cambio, si bien estuvo estrechamente ligada a las instancias de planificación política sobre la enfermedad, la actividad científica adquirió un desarrollo que excedió la mera función de asistencia técnica, y logró mantener una importante autonomía en la planificación temática de las investigaciones.

El proceso de desarrollo de la actividad científica que tuvo lugar desde fines de la década de 1960, y que se cristalizó a comienzos de los años 1970, estuvo acompañado por importantes cambios cognitivos, disciplinarios e institucionales en la producción de conocimiento científico con respecto a la década anterior. En el plano estrictamente cognitivo, la irrupción de la bioquímica y de la inmunología implicó un desplazamiento en el nivel de análisis que dio lugar, a partir de la década de 1960, y fundamentalmente a partir de 1970, a un proceso de 'molecularización' de los problemas de investigación. ${ }^{16}$ Con sus paradigmas conceptuales y herramientas técnicas, estos enfoques replantearon muchas preguntas acerca de la enfermedad en términos de la fisiología y la bioquímica del parásito (e incluso de los vectores), y de la reacción inmune del cuerpo humano ante la infección. El T. cruzi se constituyó, así, en el principal objeto de investigación, y a través de su estudio se intentó responder desde cómo eliminar al parásito mediante una droga hasta por qué algunas personas desarrollan la enfermedad y otras no (y cómo evitar que esto suceda).

En el plano de la organización social de la actividad científica, el tema fue adoptado e impulsado por algunos de los investigadores pertenecientes a la tradición de investigación biomédica, lo que marcó la inserción de la enfermedad dentro de los círculos de mayor prestigio del país ${ }^{17}$, y por los que gozaban de mayores posibilidades para su reproducción (en términos de condiciones de trabajo, acceso a financiamiento, becas etc.). Esto implicó que, en el plano institucional, la producción de conocimiento sobre la enfermedad de Chagas dejara de estar estrechamente vinculada a las instituciones creadas durante la década de 1960, y se instalara en los espacios de investigación académica. Inclusive, la influencia de este movimiento fue más allá: en los propios institutos dedicados a la enfermedad (como el Fatala Chabén) se comenzaron a desarrollar este tipo de investigaciones, bajo el influjo de los grupos liderados por los investigadores académicos (Segura, 2002; Indiech, 1995).

Si bien el principal argumento en el que se basó el apoyo a la investigación científica fue el proyecto de desarrollo de drogas o vacunas, a medida que estas decisiones comenzaron a ponerse en práctica, resultaron en un estímulo casi exclusivo al desarrollo de investigaciones básicas (principalmente sobre el parásito y sobre la respuesta inmune). De hecho, en las recomendaciones y objetivos impuestos por los distintos Comités del TDR - el Programa de apoyo más importante en términos cuantitativos -, se puede observar que la planificación de actividades se fue desplazando desde el desarrollo de drogas y vacunas, en sus inicios, hacia la producción de conocimiento básico, hacia fines de la década de 1970 (TDR, 1977, 1978, 1979). 
En este sentido, la planificación política siguió la lógica de la producción de conocimientos, donde la adopción de una perspectiva 'molecular' abrió muchos nuevos interrogantes que - ante los ojos de los científicos - debían ser resueltos antes de poder esperar resultados concretos. Los interrogantes referían, a grandes rasgos, a la necesidad de conocer más acerca del metabolismo del parásito para así encontrar un punto donde interrumpir la vida de este organismo, en lo que refiere al desarrollo de drogas. $Y$ en cuanto al desarrollo de una vacuna, en conocer con más profundidad los mecanismos que disparan el proceso patógeno en los humanos infectados, y el rol de los distintos componentes del parásito en este proceso.

\section{5-2000: la autonomización de la investigación científica}

A partir de mediados de la década de 1980, se produjo una consolidación de los grupos de investigación dedicados a temas vinculados al Chagas, y al mismo tiempo, una autonomización de los espacios de toma de decisiones políticas sobre la enfermedad. Este proceso se dio en un marco de debilitamiento de las estructuras institucionales dedicadas a intervenir sobre el problema, manifestado tanto en la merma en las tareas de fumigación como en la elaboración de estadísticas sobre la incidencia de la enfermedad en el país, y asociado a un estancamiento o retroceso de la importancia de la enfermedad en el plano de las políticas públicas nacionales. Así, durante este período convivieron dos tendencias en cierto modo contrapuestas: una que reafirmó la legitimidad del Chagas como un objeto de investigación científica, y otra marcada por un creciente desinterés de instancias estatales vinculadas con la salud pública por la enfermedad, medido en términos concretos de recursos destinados para su control y atención.

A partir de la década de 1980, el auge que la enfermedad había experimentado en las décadas anteriores en las distintas políticas públicas (científicas, sanitarias, de control de vectores) comenzó a ser reemplazado por una actitud de mayor indiferencia. Esto se tradujo en la disminución de las actividades de fumigación creadas en las décadas anteriores, en el cese de la confección de estadísticas oficiales sobre la cantidad de infectados y en la escasa atención específica a la enfermedad en los centros hospitalarios. En particular, el Programa Nacional de Chagas mostró claramente los signos de una debilidad estructural, reduciendo ocho veces la cantidad de viviendas fumigadas ente fines de la década de 1980 y principios de 1990 con relación a la década anterior (Segura, 2002, p.55-68). Estas tareas fueron parcialmente cubiertas, sin embargo, por iniciativas de tipo internacional, como la Iniciativa en Salud de los Países del Cono Sur (Incosur) de la OMS. Puesta en marcha en 1991.

Por otro lado, también se interrumpió la producción de estadísticas que dieran cuenta de la prevalencia de la enfermedad. Estas cifras provenían de un análisis al ingresar al Servicio Militar Obligatorio, y fue suspendida en el año 1992 cuando éste fue abolido. Desde entonces, no existen datos que reflejen en forma confiable la incidencia de infectados en el territorio, y se desconoce por lo tanto la evolución de la enfermedad desde entonces. Pese a todo, al menos de acuerdo a los registros realizados hasta el año 1993, el total de infectados pasó de un 5,8\% en 1981 a un promedio del 4,7\% en 1987, y luego cayeron a 1,9 \% en 1993 (Segura, 2002, p.61). Por cierto, esto obedeció a diversas causas: el efecto de 
las campañas de fumigación en los años anteriores; las modificaciones en las condiciones de vida de los sectores rurales; el crecimiento de los sectores urbanos, y así la menor incidencia de las poblaciones rurales sobre el total de la población.

\section{El crecimiento de la actividad científica}

El impulso dado por los planes de promoción a la investigación sobre la enfermedad de Chagas que se iniciaron en la década de 1970 tuvo como resultado, en las décadas siguientes, un significativo y sostenido aumento de la producción de conocimiento científico vinculado al tema, que se refleja en las publicaciones indexadas en el Science Citation Index, que pasaron de alrededor de veinte anuales a principios de la década de 1980 a cerca de cien en los años 2000.

Este crecimiento implicó, además, una autonomización del espacio de investigación científica, y el abandono de la expectativa concreta de desarrollo de una droga o vacuna comenzó a dejarse de lado. Las limitaciones que surgieron en el plano cognitivo hacia fines de los años 1970 (la falta de un modelo de estudio, la teoría de la autoinmunidad de la enfermedad), las restricciones técnicas de los laboratorios públicos para la búsqueda y diseño de drogas, y el desinterés de los laboratorios privados, transformaron al desarrollo de una droga en un mero elemento retórico, pero no en un objetivo concreto de las investigaciones. De hecho, una de las principales características de las investigaciones en bioquímica y biología molecular - las disciplinas que más participan en las investigaciones - fue la adopción, durante este período, del T. cruzi como un modelo de investigación biológica válido dentro de ciertas especialidades de la biología (Parodi, 1994, p.249). Es decir, el propio tripanosoma fue adoptado como modelo de investigación a partir del cual desentrañar la naturaleza de ciertos procesos metabólicos (como la degradación de azúcares, por ejemplo), y establecer comparaciones con las evidencias surgidas de las indagaciones sobre otros organismos (hongos, bacterias o mamíferos).

En contraposición a la tendencia creciente en estas áreas, las investigaciones en otras disciplinas históricamente ligadas a la enfermedad, como la cardiología, siguieron una tendencia decreciente. En parte, esto se entiende por la baja institucionalización de la enfermedad como una especialidad de la cardiología: los enfermos de Chagas son cada vez más considerados como casos de afecciones cardíacas generales, sin resaltar su especificidad. Y esta inespecificidad de la enfermedad se refuerza en la propia práctica médica, en la medida en que no existe ningún tratamiento específico para la enfermedad en su etapa crónica (y el tema es objeto de controversia entre la comunidad de especialistas). Los pacientes chagásicos, entonces, son atendidos por cardiólogos en servicios de cardiología general, y son tratados con terapéuticas no específicas, ya sean drogas que regulen el ritmo cardíaco, ya sean tratamientos de tipo eléctrico, como marcapasos o desfibriladores, y muy incipientemente con tratamientos basados en el uso de células madre que apuntan a revitalizar el tejido cardiaco dañado.

En definitiva, el resultado de este escenario marcado por el decreciente interés por la enfermedad en el plano sanitario y el crecimiento y autonomización de la investigación científica tuvieron como rasgo fundamental en las últimas décadas una débil articulación 
entre la producción de conocimientos y las instancias de intervención concreta sobre la enfermedad. Paulatinamente, la posible utilidad de los conocimientos científicos, esgrimida como un argumento central de las políticas de promoción de las investigaciones desde la década de 1970, fue reemplazada, en la práctica, por la adopción de los criterios 'clásicos' de promoción y evaluación de la investigación: la productividad (cantidad y calidad de las publicaciones, según el número de artículos y el factor de impacto de las revistas), y el cumplimiento de las funciones asignadas a los distintos roles profesionales (ya sean éstas tareas de docencia, atención a pacientes, o desarrollo de tareas de diagnóstico, dependiendo de la institución).

\section{Representaciones, conocimientos e interacciones: la trayectoria de la enfermedad de Chagas en Argentina}

El análisis histórico de la forma en que la enfermedad de Chagas se ha configurado como problema científico y social en Argentina, así como el de las representaciones y de los conocimientos científicos movilizados en cada momento, nos muestra diferentes configuraciones a lo largo del siglo XX. Así, la enfermedad fue considerada, a lo largo de su historia, como una patología inexistente, como una 'rareza', y luego como un problema nacional de primera envergadura. Al mismo tiempo, pasó de ser entendida como un problema de vivienda rural a ser un problema de la logística necesaria para desinfectar las viviendas. Hacia mediados del siglo XX, la connotación rural fue complementada con la idea de que se trataba también de un problema urbano y de una limitación concreta en la fuerza de trabajo (y por ende de desarrollo) del país. Se observó entonces una implicación directa del Estado, cuya preocupación fue, entonces, no sólo identificar y cuantificar a los enfermos, sino poder curarlos. Y cuando hubo tratamientos disponibles, el principal interés se centró en el reemplazo de esas drogas por otras más efectivas o por vacunas que prevengan la enfermedad.

Las modificaciones en las representaciones de la enfermedad no pueden concebirse como el reflejo de un proceso continuo y acumulativo en los saberes acerca de la enfermedad. Sin duda, están basadas en la interpretación de ciertos elementos - y de las relaciones entre ellos - a los que sí podríamos considerar objetivos: vinchucas, parásitos, alteraciones cardiacas, músculos atrofiados y lesiones nerviosas se presentan, ante nuestros ojos, como evidencias de difícil contrastación (Gusfield, 1981). Sin embargo, es posible encontrar en la historia elementos que nos muestran que los enunciados acerca de la enfermedad no son, ni mucho menos fueron cuestiones inalterables. De hecho, los datos provenientes del mundo científico, tras su apariencia objetiva e inamovible son, en realidad, cambiantes, sus significados flexibles, y su aceptación negociada entre distintos actores. Esto se puede ver en la redefinición profunda que tuvo el significado de la enfermedad en el plano cognitivo: ciertos hechos fueron descartados, como la relación con el bocio y el cretinismo, aun cuando esto era una parte central de la descripción de la enfermedad que había hecho su 'descubridor', Carlos Chagas. Mientras que otros hechos en un principio menores, como las alteraciones cardiacas, adquirieron una importancia central en la reconfiguración de la enfermedad como una dolencia crónica. Y aun así, la importancia de la relación 
entre el parásito y las lesiones cardiacas se desdibuja en la actualidad en la atención a los enfermos, a quienes se los trata de igual manera que a cualquier otro enfermo cardiaco.

De un modo más evidente se presentan, en cambio, los cambios sociales y políticos que llevaron a que ciertas representaciones sean aceptadas y otras rechazadas, que los recursos se destinen hacia ciertas posibles formas de intervención (que muchas veces resultan impracticables) y no otras. Ha sido una intención del trabajo, entonces, poner en relación estas distintas dimensiones, buscando las huellas de las circunstancias que jalonaron la trayectoria de la enfermedad, de sus altos y bajos en la consideración social y política y, en definitiva, de su actual ubicación en plano de la salud pública de Argentina y del continente.

\section{NOTAS}

${ }^{1}$ En este sentido, Rosenberg y Golden (1992, p.XIII) señala que "la enfermedad es al mismo tiempo un evento biológico, un repertorio de construcciones verbales específicos que reflejan la historia intelectual e institucional de la medicina en una generación determinada, la posibilidad de una política pública y la legitimación de esa política, un aspecto del rol social y una entidad individual intrafísica, una sanción para valores culturales, y un elemento estructurante de la relación médico-paciente".

${ }^{2}$ La 'etapa aguda' refiere al momento inicial de la enfermedad, donde la infección con el parásito promueve un tipo de reacciones en el cuerpo humano. Cuando estos síntomas iniciales desaparecen, la enfermedad entra en su 'etapa crónica', en el que las consecuencias son de más largo plazo. Los síntomas atribuidos a cada una de estas etapas han ido variando a lo largo de la historia.

${ }^{3}$ Las investigaciones de Kraus no negaban la existencia de la enfermedad en su etapa aguda, entidad poco estabilizada en el momento, ya que no se había desarrollado evidencia sobre el tema, aunque luego sus afirmaciones fueron tomadas en ese sentido por parte de la comunidad médica brasilera opuesta a Chagas (ver Kropf, 2006).

${ }^{4}$ Guerreiro y Machado eran investigadores del Instituto Oswaldo Cruz, que adaptaron el método desarrollado por Wasserman para la sífilis (reacción de fijación del complemento) a la enfermedad de Chagas. La baja especificidad implicaba que el resultado positivo de la prueba se debía, en muchos casos, a la presencia de otros organismos patógenos, y no necesariamente al T. cruzi.

${ }^{5}$ Este método consistía en transfundir sangre supuestamente infectada a animales sensibles al parásito (cobayos, ratas, conejos, perros). Si luego éstos presentaban signos del parásito, se consideraba el caso positivo.

${ }^{6}$ El xenodiagnóstico fue adaptado por Émille Brumpt, destacado parasitólogo francés, y consistía en exponer a la supuesta persona infectada a la picadura sucesiva de insectos vectores no infectados. Luego, si los insectos mostraban el parásito en su interior, podía establecerse que el individuo estaba infectado.

${ }^{7}$ La enfermedad de Chagas ha sido un tema poco abordado por la historia de la ciencia local. Existen algunos trabajos hagiográficos centrados en la figura de Mazza (Sierra Iglesias, 1990; Ivern, 1987), y una muy escasa producción historiográfica (Lorenzano, 2003; Zabala, 2007).

${ }^{8}$ Para estudios sobre la enfermedad de Chagas en Brasil, incluyendo las controversias sobre el tema, ver Benchimol, Teixeira, 1993, Coutinho, 1999, Coutinho, Freire Jr., Dias, 1999, Kropf, Azevedo, Ferreira, 2003 y Kropf, 2006.

${ }^{9}$ Paradójicamente, fueron Emmanuel Dias y Evandro Chagas quienes propusieron la denominación del "Síndrome de Romaña" o "Sinal de Romaña" en la XIX Reunión de la Sociedad Argentina de Patología Regional del Norte, en 1935, reconociendo así la originalidad de las formulaciones de Romaña, que daban un significado novedoso a algunas de las afirmaciones de Carlos Chagas (ver Dias, 1939; Zabala, 2007)

${ }^{10}$ Según Sierra Iglesias (1990, p.342) fue Rodolfo Tálice, profesor de parasitología de la Universidad de Montevideo e investigador de la enfermedad de Chagas en Uruguay, quien primero propuso en el VI Congreso Nacional de Medicina (Córdoba, Argentina, 1938) que la enfermedad debería llamarse 'enfermedad de Chagas-Mazza'. 
11 Por cierto, procesos similares se vivieron en otros países, sobre todo en Brasil, donde en el mismo período la enfermedad fue también reconocida como un hecho científico y como un problema social (ver Coutinho, 1999; Kropf, Azevedo, Ferreira, 2003; Kropf, 2006).

${ }^{12}$ La reconversión de la enfermedad en una dolencia crónica cardiaca es analizada en los mencionados trabajos de Kropf, Azevedo, Ferreira, 2003, Kropf, 2006 y Perletth, 1997.

${ }^{13}$ Sobre el proceso de reconocimiento de la enfermedad en Argentina, la elaboración de estadísticas y la relación con la política sanitarista de Perón, ver Zabala, 2007 y en prensa.

${ }^{14}$ Para un análisis del impacto del gobierno de Juan Perón en distintas esferas de la sociedad ver Torre, 2002. Sobre las reformas en el área de salud, ver Belmartino, 2005, Rammaciotti, 2005, Carbonetti, González Leandri, 2008.

${ }^{15}$ Entre 1955 y 1972 se sucedieron siete presidentes, de los cuales sólo dos de ellos fueron elegidos en forma democrática (aunque con el peronismo prohibido): Arturo Frondizi (1958-1962) y Arturo Illia (19631966). Ambos fueron derrocados por sendos golpes de Estado en manos de los militares.

${ }^{16}$ El uso de la idea de 'molecularización' está relacionada con el enfoque propuesto en de Charadevian et al. (1999). Así, el concepto no se reduce a la introducción de la biología molecular, sino que refiere "al largo proceso histórico que llevó a médicos e investigadores en biología y medicina a concentrar en las moléculas la búsqueda de la solución a todos sus problemas clínicos y de investigación". Este proceso excedió el ámbito argentino, y para el caso de Brasil fue analizado en Coutinho, 1999.

${ }^{17}$ A la que pertenecían los Premios Nobel Bernardo Houssay (1947) y Luis Leloir (1970).

\section{REFERENCIAS}

BELMARTINO, Susana.

La atención médica argentina en el siglo XX: instituciones y procesos. Buenos Aires: Siglo XXI Editores Argentina. 2005.

BENCHIMOL, Jaime L.; TEIXEIRA, Luiz

Antonio.

Cobras, largatos e outros bichos: uma história comparativa dos Institutos Oswaldo Cruz e Butantan. Rio de Janeiro: Fiocruz; Editora da UFRJ. 1993.

CARBONETTI, Adrián; GONZÁLEZ LEANDRI, Ricardo (Org.).

Historias de salud y enfermedad en América Latina, siglos $X I X$ y XX. Córdoba: Universidad Nacional de Córdoba. 2008.

CARRILLO, Ramón.

Progreso de la medicina sanitaria en la República Argentina: conferencia pronunciada el 21 de febrero de 1951 en el aula magna del Colegio Nacional de Buenos Aires, en el ciclo Recientes Conquistas de la Sanidad Argentina. In: Carrillo, Ramón. Contribuciones al conocimiento sanitario: obras completas II. Buenos Aires: Eudeba. 1974.

CHAGAS, Carlos.

Estado atual da tripanossomíase americana.

Revista de Biologia e Higiene, São Paulo, v.5, n.2, p.58-64. 1934.

COUTINHO, Marilia.

Ninety years of Chagas disease. Social Studies of Science, London, v.29, n.4, p.519-550. 1999.
COUTINHO, Marilia; FREIRE JR., Olival; DIAS, João Carlos Pinto.

The Nobel enigma: Carlos Chagas' nominations for the Nobel Prize. Memórias do Instituto Oswaldo Cruz, Rio de Janeiro, v.94, supl.1, p.123-129. 1999.

DIAS, Emmanuel.

O sinal de Romaña e sua influência na evolução dos conhecimentos sobre a moléstia de Chagas. Brazil Medico, Rio de Janeiro, v.53, n.42, p.965-970. 1939.

DIAS, Emmanuel; LARANJA, Francisco; NÓBREGA, Genard.

Doença de Chagas. Memórias do Instituto Oswaldo Cruz. Rio de Janeiro, v.43, n.3, p.495-581. 1945.

DIAS, Emmanuel; PELLEGRINO, J.

Alguns ensaios com o gammexane no combate aos transmissores da doença de Chagas, Brazil Medico. Rio de Janeiro, v. 62, n.18-20, p.185-191. 1948.

FONZO GANDOLFO, C.

Clínica de enfermedades infecciosas y su tratamiento. t.2. 1.ed. Buenos Aires: Aniceto López Ed. 1936.

GUHL, Felipe; LAZDINS-HELDS, Janis K. (Ed.).Reporte del grupo de trabajo científico sobre la enfermedad de Chagas. Grupo de trabajo científico 17-20 de abril de 2005. Actualizado en julio de 2007, Buenos Aires, Argentina. Washington: World Health 
Organization on behalf of the Special Programme. TDR/SWG/09. 2007.

GUSFIELD, Joseph R.

The culture of public problems: drinking-driving and the symbolic order. Chicago: The University of Chicago Press. 1981.

INDIECH.

Instituto Nacional de Chagas. Nuestra memoria histórica: 1962-1995. Buenos Aires: Indicech. 1995.

IVERN, Andrés.

Vida y obra de Salvador Mazza. Santa Fe: Editorial Nuevo Rumbo. 1987.

KRAUS, Rudolf.

El Instituto Bacteriológico del Departamento Nacional de Higiene y su labor. Anales del Departamento Nacional de Higiene, Buenos Aires. 1920.

KRAUS, Rudolf; MAGGIO, C.; ROSENBUSCH, F. Bocio, cretinismo y enfermedad de Chagas: segunda comunicación. La Prensa Médica Argentina, Buenos Aires, año 3, n.17, p.177-180. 1916.

KRAUS, Rudolf; MAGGIO, C.; ROSENBUSCH, F. Bocio, cretinismo y enfermedad de Chagas. La Prensa Médica Argentina, Buenos Aires, año 2, n.1, p.1-5. 1915.

KROPF, Simone.

Doença de Chagas, doença do Brasil: ciência, saúde e nação (1909-1962). Tesis (Doctorado) Programa de Pós-Graduação em História, Universidade Federal Fluminense, Niterói. 2006.

KROPF, Simone; AZEVEDO, Nara; FERREIRA, Luiz Otávio.

Biomedical research and public health in Brazil: the case of Chagas' disease (1909-1950), Social History of Medicine, Oxford, v.16, n.1, p.111-129. 2003.

LARANJA, Francisco; DIAS, Emmanuel; NÓBREGA, Genard.

Clínica e terapêutica da doença de Chagas. Memórias do Instituto Oswaldo Cruz, Rio de Janeiro, n.46, v.2, p.473-529. 1948.

LORENZANO, Cesar.

La enfermedad de Chagas-Mazza, In:

Lorenzano, Cesar. (Ed.). Historias de la ciencia argentina. Buenos Aires: Eduntref. p.181-207. 2003.

\section{LOZANO, N.}

Reseña histórica del Instituto Bacteriológico. Anales del Departamento Nacional de Higiene, Buenos Aires. 1920.

MAZZA, Salvador.

Sobre el desacierto de adoptar un subtítulo para la enfermedad de Chagas. La Prensa Médica Argentina, Buenos Aires, año 30, n.10, p.433-440. 1943.

MAZZA, Salvador

La enfermedad de Chagas en la República Argentina. In: Congreso Nacional de Medicina, 6., 16-21 oct. 1938, Córdoba. Actas y trabajos.... t.3. s.l: s.n. p.9-20. 1939.

MAZZA, Salvador.

Observación de infección espontánea del perro por el Schyzotripanum cruzi. Revista de la Universidad de Buenos Aires, Buenos Aires, sección IV, tomo IV, p.378-392. 1926.

MAZZA, Salvador ; ROMAÑA, Cecilio; SCHURMANN, K.

Nuevas observaciones sobre la infección espontánea de armadillos del país por el Trypanosoma cruzi. Publicaciones de la Mepra, Buenos Aires, n.3. 1931.

MAZZA, Salvador; RUCHELLI, A.

Comprobación de dos casos de enfermedad de Chagas en Tinogasta (Catamarca). Publicaciones de la Mepra, Buenos Aires, n.20, p.3-19. 1934.

\section{MÜLHENS, $\mathrm{P}$}

Comunicación preliminar sobre parásitos sanguíneos en el norte argentino. Revista de la Asociación Médica Argentina, sesiones de la Sociedad Argentina de Biología, t.37, p.205-220. 1924.

PARODI, Armando J.

Serendipity, or how working with glycoproteins from Trypanosomatids changed my life (with a little help from my friends). Ciência e Cultura, Journal of the Brazilian Association for the Advancement of Science, São Paulo, v. 46, n.4, p.249-254. 1994.

PERLETH, Matthias.

Historical aspects of American trypanosomiasis (Chagas' disease). Medizin in

Entwicklungslandern. Bd.43. Frankfurt: Peter Lang Publishing. 1997.

RAMACCIOTTI, Karina Inés.

Las huellas eugénicas en la política sanitaria Argentina (1946-1955). In: Miranda, M.; Vallejo, G. (Ed.). Darwinismo social y eugenesia en el mundo Latino. Buenos Aires: Siglo XXI Editores Argentina. p.311-350. 2005.

ROMAÑA, Cecilio.

Discurso del representante del Comité Organizador y Director de la Dirección de Lucha Contra la Enfermedad de Chagas, dr. Cecilio Romaña. In: Conferencia Nacional de Enfermedad de Chagas, 1., 25-27 jan. 1953, Buenos Aires. Primera Conferencia.... Buenos Aires: Ministerio de Asistencia Social y Salud Pública de la Nación. p.9-11, 1953. 
ROMAÑA, Cecilio

Panorama continental de la enfermedad de Chagas. In: Congresso Brasileiro de Higiene, 9., 1951, Porto Alegre. Anais... Porto Alegre: s.n. p.209-215. 1951.

ROMAÑA, Cecilio.

Acerca de un síntoma inicial de valor para el diagnóstico de forma aguda de la enfermedad de Chagas: la conjuntivitis esquizotripanósica unilateral (hipótesis sobre puerta de entrada conjuntiva de la enfermedad). Publicaciones de la Mepra, investigaciones sobre la enfermedad de Chagas, Buenos Aires, n.22, p.16-28. 1935.

ROMAÑA, Cecilio.

Comprobación de formas agudas de tripanosomiasis americana en el Chaco Austral y santafesino. Publicaciones de la Mepra, investigaciones sobre la enfermedad de Chagas, Buenos Aires, n.14, p.21-24. 1934.

ROMAÑA, Cecilio; ÁBALOS, J.W.

Acción del 'gammexane' sobre los triatomideos: 'control' domiciliario. Anales del Instituto de Medicina Regional, Tucumán, v.2, n.2, p.95-106. 1948.

ROSENBERG, Charles E.; GOLDEN, Janet. (Ed.). Framing disease: studies in cultural history. New Jersey: Rutger University Press. 1992.

ROSENBUSCH, F.; MAGGIO, C.

'Schizotrypanum cruzi' en la República Argentina. Archivos de Higiene, Buenos Aires, t.5, p.123-125. 1912.

SEGURA, Elsa L.

El control de la enfermedad de Chagas en la República Argentina. In: Silveira (Ed.). El control de la enfermedad de Chagas en los países del Cono Sur de América: historia de una iniciativa internacional. Buenos Aires: Organización Panamericana de la Salud. 2002.
SIERRA IGLESIAS, Jobino Pedro.

Salvador Mazza, redescubridor de la enfermedad de Chagas: su vida, su obra. San Salvador de Jujuy: Universidad Nacional de Jujuy. 1990.

SILVEIRA, Antonio Carlos.

Situação do controle da transmissão vetorial da doença de Chagas nas Américas. Cadernos de Saúde Pública, Rio de Janeiro, v.16, supl.2, p.35-42. 2000.

\section{STEPAN, Nancy.}

Beginnings of Brazilian science: Oswaldo Cruz, medical research and policy, 1890-1920. New York: Watson Publisher International. 1981.

TORRE, Juan Carlos (Dir.).

Los años peronistas: 1943-1955. Buenos Aires: Sudamericana. (Colección Nueva Historia Argentina, v.8). 2002.

WORBOYS, Michael.

Tropical diseases; En: Bynum, WF; Porter, Roy (Ed.). Companion encyclopaedia of the history of medicine. London: Routledge. p.512-536. 1993.

YORKE, Warrington.

Chagas' disease: a critical review. Tropical

Diseases Bulletin, London, v.34, n.4, p.275-300, 1937.

ZABALA, Juan Pablo.

Producción y uso de conocimientos científicos vinculados a la enfermedad de Chagas, Argentina, 1915-2000. Tesis (Doctorado) Facultad Latinoamericana de Ciencias Sociales; Université Paris 1- Panthéon, Sorbonne. 2007.

ZABALA, Juan Pablo.

La enfermedad de Chagas como 'problema social nacional' en Argentina: populismo, sanitarismo e investigación científica. In: Palmer, S.; Di Liscia, M.S.; Hochman G. (Ed.). Patologias de la patria: enfermedades y enfermos en América Latina (XVIII-XX). Buenos Aires: Editorial Norma. en prensa.

\section{$\rightarrow \rightarrow \rightarrow<<$}

